\title{
Stimulus-recognition and response-recall dependency in paired-associate learning
}

\author{
MARY E. GRUNKE and JAMES V. HINRICHS \\ University of Iowa, Iowa City, Iowa 52242
}

\begin{abstract}
To test the hypothesis that overt associative responding may influence concurrent stimulus recognition decisions while controlling the problem of disproportionate representation of particular learning stages and items, two groups of subjects were given three trials on a paired-associate task. On a final test trial, one group made only stimulus recognition responses, while a second group made both stimulus recognition and associative matching responses. No differences between the groups were found, consistent with the notion of two methodologically separable stages of paired-associate learning, pre- and poststimulus recognition. Previous findings were replicated regarding (a) the dependency of associative recall upon stimulus recognition in paired-associate learning, (b) the role of the first letter in recognition of CCCs, and (c) the effect of M-value on correct recognition and on false recognition.
\end{abstract}

Recent interest in stimulus coding has resulted in concern with the role of stimulus recognition in associative learning. Retrieval from memory of acquired associative responses may be viewed as consisting of two steps: (a) a stimulus coding stage involving linkage of the overt stimulus with some representation of its previous occurrence, and (b) an associative step requiring elicitation of the proper associative response. The second stage is contingent upon the first. Several investigators have attempted to identify the loci of various effects in paired-associate learning (e.g., word-picture differences and imagery-instruction effects) by having subjects make both stimulus recognition and associative responses to each tested item (Bower, 1970; Peterson \& McGee, 1974; Peterson \& Murray, 1973; Wicker, 1970). Recognition performance presumably reflects progress in the stimulus coding stage, and correct responses (apart from chance guessing) indicate completion of both stages.

The assertion that stimulus recognition is a prerequisite for correct-response recall in paired-associate learning is generally accepted as intuitively reasonable and empirically sound (e.g., Martin, 1967a, 1967b). The main purpose of the current study was to scrutinize particular procedures used to verify that generalization, to see whether a recognition response of "old" can validly be interpreted as reflecting prior stimulus-code reinstatement, the dividing point in a two-stage analysis of paired-associate learning. Specifically, the purpose of the experiment was (a) to examine the hypothesis that overt associative responding may influence decision criteria in stimulus recognition judgments when recognition and associative responses are made cotemporally in paired-associate tasks and (b) to measure the probability of a correct associative

This paper is based on a master's thesis completed by the first author under the direction of the second author. Requests for reprints should be sent to Mary E. Grunke, Department of Psychology, University of Iowa, Iowa City, Iowa 52242. response given stimulus nonrecognition, $\mathrm{P}(\mathrm{CR} / \mathrm{NR})$, in a way that reduces distortion due to disproportionate representation of particular stages of learning and items.

When subjects must identify an item as old or new and, at the same time, try to recall a response to that item, the two decisions might not be made independently of each other. A subject may base his recognition decision in part on his ability to recall a response, reasoning, "If I do not know what response goes with an item, it must be new." Specifically, it was hypothesized that when a subject doubts the correctness of his associative response, he uses a more stringent decision criterion in assessing the familiarity of the given stimulus. When recognition performance is evaluated as if a subject's criterion point were static, measures of stimulus familiarity become confounded with associative-response confidence. The tendency to report nonrecognition when the associative match cannot be recalled seems, in fact, to be a sensible strategy when stimulus frequency and pair frequency closely covary, as is the case in most paired-associate experiments.

In the present experiment two groups of subjects were given study and test trials on paired-associate lists containing both high- and low-M CCCs. At a given point in learning, stimulus recognition was tested with one group making both stimulus recognition and associative responses and the second group giving only recognition responses. Recognition performance of the two groups was compared to determine whether the requirement that subjects make associative responses with each stimulus-recognition response changes recognition performance.

When the $\mathrm{P}(\mathrm{CR} / \mathrm{NR})$ is at chance guessing level, the hypothesis that stimulus recognition is a precondition for associative recall is supported. Whenever data involving contingencies are examined, however, some experimental conditions, list items, and subjects contribute disproportionately to measurements, leading 
to possible distortion of results. Such is likely to be the case when the $P(C R / N R)$ is measured over a series of trials. A large proportion of stimulus nonrecognitions occur during the early stages of learning, the number of nonrecognition in late trials being, in fact, very small. If data are pooled over trials to estimate the overall $\mathrm{P}(\mathrm{CR} / \mathrm{NR})$, earlier trials are overrepresented in comparison to later trials. Response recall, regardless of stimulus recognition, is poorer in early trials. Recall may appear contingent on stimulus recognition, then, merely because most instances of stimulus nonrecognition occur on early trials. The current experiment assessed the $\mathrm{P}(\mathrm{CR} / \mathrm{NR})$ only at one point in learning, a point where associative recall was above chance level and recognition performance had not yet reached a ceiling.

\section{METHOD}

\section{Materials}

Four 14-item lists of CCC-digit pairs were constructed with half the stimulus terms in each list being of high $M$ and haif of low M. Low-M trigrams had an association value of $4 \%, 8 \%$, or $13 \%$; the association value of high-M trigrams was $75 \%, 79 \%$, or 83\% (Witmer, 1935). The four lists represented the factorial combination of two sets of CCCs and two response assignments. Responses, the digits one through seven, were randomly assigned to the stimuli with the restriction that each digit occur exactly once per list per M condition (high or low). Two distractor item sets were composed of seven high-M and seven low-M CCCs. Each group of distractor items was used with a given stimulus set.

\section{Procedure and Design}

The items were presented with a Kodak Carousel 750 projector. Subjects made responses by pressing one of nine buttons labeled with the digits one through seven and " $O$," for old, and " $\mathrm{N}$," for new.

In the first part of the experiment, all subjects followed the same procedure. Three study and three recall trials were given in alternation, beginning with a recall trial to familiarize subjects with the response procedure. Three successive slides showing a trigram, then a digit, and then an empty dark field comprised each pair presentation. On test trials each CCC was presented in alternation with slides showing "Number?" As each trigram appeared, subjects were to decide which labeled button to press and then were to press that button when "Number?" was shown. Subjects were told to make a response to each item, guessing when uncertain of the correct digit. The slide presentation rate was $2.5 \mathrm{sec}$; thus, $7.5 \mathrm{sec}$ was allotted per pair per study trial and $5 \mathrm{sec}$ per item per test trial.

After the third study trial, revised instructions were given. In the final test trial, stimulus trigrams interspersed randomly with distractor items were shown, each followed by a slide with "Old or New?". When the slide "Old or New?" appeared, the subject was to indicate whether he recognized the trigram or not by pressing either the "O" or "N" button. For half the subjects, "Number?" followed each "Old or New?" slide. As in previous trials, the "Number?" slide indicated the subject was to press the correct number-labeled button, guessing a button if the response was unknown or if the trigram seemed new. Again slides were presented every $2.5 \mathrm{sec}$, allowing $5 \mathrm{sec}$ per item for the recognition-only group and $7.5 \mathrm{sec}$ for the recognition-and-recall group.

Ten subjects were randomly assigned to each of eight conditions representing the factorial combination of four lists and two final-test response requirements. The 80 college sophomores, recruited from the elementary psychology course at the Lniversity of Iowa, were tested in groups of 1 to 5 members per session.

\section{RESULTS}

\section{Associative Performance: Trials 1-3}

The probability of a correct response on Trial 1, i.e., before the first pair presentation, was .144 , excluding the data of 11 subjects who responded less than half the time on the first trial. This value is virtually equivalent to .143 , the expected rate given seven equiprobable response alternatives and random guessing. The recognition-only and recognition-and-recall groups, which were treated identically through Trial 3 , showed statistically equivalent performance on Trials 2 and 3 , $\mathrm{F}<1$.

\section{Final Trial Performance}

Recognition performance. Final trial recognition performance was evaluated by tabulating for each subject the mean number of correct recognitions of stimulus terms and the mean number of correct rejections of distracter CCCs. Counter to expectations, two analyses of variance, one on each performance measure, indicated no main effect of final-trial response requirements, ps $>.10$, and no interactions of this factor with list variables or $\mathrm{M}$, all ps $>.04$. High-M items were more often correctly recognized as old, $[F(1,72)=$ $49.28, \mathrm{p}<.001]$, and correctly identified as new $[F(1,72)=60.60, p<.001]$ than were low-M items. The mean proportion of correct recognitions was .86 for low-M, and .97 for high-M items. Low-M distracter items were correctly rejected with a probability of .74 and high-M items witha .89 probability. No interactions of $M$ with list variables reached significance, all ps $>.01$.

Recall/recognition contingency: Stimulus items. Correct associative responses were given to 260 of the 509 stimulus items correctly recognized by the recognition-and-recall group. Of 50 incorrect recognition responses of "new," 6 were followed by correct associative recall. Thus, the $\mathrm{P}(\mathrm{CR} / \mathrm{NR})$ was .120 , a value clearly attributable to chance.

To control for unequal representation of easy and difficult items in the computation of conditional probabilities, the final trial responses of each subject were grouped according to the number of prior correct associative responses-zero, one, or two-given to each item. The final-trial correct-associative-response probabilities conditional upon stimulus recognition performance for each category of item difficulty (i.e., zero, one, or two prior correct responses) are presented in Table 1. When stimuli were correctly recognized, the probability of a correct associative response increased with the number of preceding correct responses. Given nonrecognition, however, correct-associative-response probability remained at chance level regardless of prior response histories. 
Table 1

Proportion Correct Associative Responses (CR) in Final Trial Given CR History and Stimulus Recognition (R) or Nonrecognition (NR)

\begin{tabular}{lrrr}
\hline & \multicolumn{3}{c}{ CR History } \\
& \multicolumn{1}{c}{ (Number of Preceding CRs) } \\
\cline { 2 - 4 } & 0 & 1 & 2 \\
\hline P(CR/CR History \& R) & .316 & .508 & .803 \\
N & 196 & 181 & 132 \\
P(CR/CR History \& NR) & .138 & .067 & .167 \\
N & 29 & 15 & 6 \\
\hline
\end{tabular}

Recall/recognition contingency: Distracter items. Following Martin (1968) an analysis was made of recognition and recall responses to distracter CCCs. An associative response was scored "correct" if it matched the digit paired on previous trials with a stimulus item beginning with the same letter as the given distracter CCC. Each distracter CCC had no more than one possible "correct" response. When a distracter item was labeled "old," the probability of a "correct" response was .33 (based on 94 observations), whereas given "new" recognition responses, correct response probability was .17 (244 observations). This difference in response probability is significant at the .001 level, $z=$ 3.13 .

\section{DISCUSSION}

Results showed no evidence of a dependence of recognition responding upon concurrent associative responding. While an alternate conclusion is not precluded, in view of the substantial experimental power and adherence to past procedures it is at least unlikely that the postulated methodological artifact has generally been operative in previous studies. Further, there was no support for the assertion that the method of separating paired-associate learning into two stages-pre- and poststimulus recognition-by having subjects make concurrent stimulus recognition and associative recall responses is not viable.

This study replicated previous findings that (a) the probability of retrieving a correct associative response is at chance level when the stimulus is not recognized even when unequal contributions to conditional probabilities of particular learning stages and items is controlled; (b) the stimulus-recognition prerequisite for association activation depends largely upon the first letter of CCC stimuli and, given stimulus recognition on this basis, appropriate associations are activated (Margin, 1968); and (c) correct recognition of consonant trigrams is directly related to M-value, while false recognition varies inversely with $M$ (Martin \& Melton, 1970).

\section{REFERENCES}

Bower, G. H. Imagery as a relational organizer in associative learning. Journal of Verbal Learning and Verbal Behavior, $1970,9,529-533$.

Martin, E. Relation between stimulus recognition and paired-associate learning. Journal of Experimental Psychology, $1967,74,500-505$.

Martin, E. Stimulus recognition in aural paired-associate learning. Journal of Verbal Learning and Verbal Behavior, 1967b, 6 , 272-276.

Martin, E. Recognition and correct responding mediated by first letter of trigram stimuli. Journal of Verbal Learning and Verbal Behavior, 1968, 7, 703-704.

Martin, E., \& Melton, A. W. Meaningfulness and trigram recognition. Journal of Verbal Learning and Verbal Behavior. 1970, 9, 126-135.

Peterson, M. J., \& McGee, S. H. Effects of imagery instructions, imagery ratings, and number of dictionary meanings upon recogntion and recall. Journal of Experimental Psychology, 1974, 102, 1007-1014.

Peterson, M. J., \& Murray, A. Enhancement of items and associations. Joumal of Experimental Psychology, 1973, 101, 82-89.

Wicker, F. W. On the locus of picture-word differences in paired-associate learning. Journal of Verbal Learning and Verbal Behavior, 1970, 9, 52-57.

Witmer, $L$. R. The association value of three-place consonant syllables. Journal of Genetic Psychology, 1935, 47, 337-360.

(Received for publication January 27, 1975.) 